\title{
Eradication of endemic syphilis in Bosnia
}

\author{
N ARSLANAGIĆ, M BOKONJIĆ, K MACANOVIĆ \\ From the Department of Dermatology, University of Sarajevo, Sarajevo, Yugoslavia
}

SUMMARY The control of syphilis in Bosnia was organised and started by Professor Ernest Grin in 1948 and continued to the end of 1951. Regardless of the clinical picture, the treatment of endemic syphilis consisted of an oil suspension of penicillin with aluminium monostearate (PAM) 3.6 to $4 \cdot 2$ MIU for adults in six or seven intramuscular injections of $0.6 \mathrm{MIU}$ every day or $1.2 \mathrm{MIU}$ every other day. Today endemic syphilis in Yugoslavia is only historic fact.

The first written data on endemic syphilis in Bosnia were given by Dr Leopold Glück, a doctor in Foča.' Exact data on the origin of endemic syphilis in Bosnia, however, are not known. One hypothesis is that it was introduced by Turkish soldiers, specially along routes or in borderline settlements. The second hypothesis, based on the name "franka" ("frenjak") indicates the possibility that the disease was introduced by French sailors during the occupation of 1809 . This hypothesis is supported by the fact that endemic foci of the disease in Croatia were observed simultaneously. The interesting fact is that "morbus Skerljevo", named after the village Skerljevo, corresponded to syphilis and that the villagers had participated in the Turkish war on the side of the emperor Josif in 1781.2

Data from Bosnian monks indicated that syphilis was known in Bosnia in 1834 (Libellius Medicinalis) and in 1850 (Prijatelj Bosanski), though Fleger gave evidence that the disease emerged at the end of the eighteenth century. ${ }^{3}$ It seems that even if the disease was common in the Moslem population, it cannot be linked with the Turkish occupation or any other historic event. ${ }^{4}$ There are no data on the incidence of syphilis in Bosnia and Hercegovina during the Turkish occupation. During the Austrian occupation, however, the disease was endemic and many people were affected, especially in the regions of Cazin and central and east Bosnia. The lowest incidence was in Hercegovina. Data from 1885-1911 indicate that 41000 patients $(8.3 \%$ of the total number examined) had active disease and 31000 of these patients had tertiary syphilis. ${ }^{5}$ Data on latent syphilis were not available.

Address for reprints: Dr N Arslanagic, Department of Dermatology, University of Sarajevo, Sarajevo, Yugoslavia.

Accepted for publication 1 May 1988
In 1927-33 an important control programme was taken in the fight against endemic syphilis in Bosnia and Hercegovina. During this period many cases of latent syphilis were discovered: out of 60000 patients infected, 18000 had early, 7000 tertiary, and 35000 latent disease. Out of the total population examined, $11.8 \%$ were infected. ${ }^{46}$

It is worth mentioning that the quisling government of the independent state of Croatia, using the Jewish doctors taken from concentration camps, tried to campaign against endemic syphilis in 1941-2, but without success. It is understandable that during the second world war, because of fluctuations of the population and extremely low standards of hygiene, many new endemic areas of syphilis appeared.

\section{Action}

There were numerous reasons for the failure of the above actions against endemic syphilis in Bosnia. The campaigns were not carried on in the whole endemic area, and there were many interruptions during the course of the campaign. Systematic examinations never included the whole population, and many infected people, especially those recently infected, were not detected. Epidemiological factors and principles were not used sufficiently, although they played a major part in the efforts to eradicate this disease. Available chemotherapeutic drugs were not appropriate for fieldwork, especially in the remote areas. Extremely bad social, economic, and health standards, as well as low cultural levels predisposed to syphilis persisting as endemic disease. ${ }^{6}$

Some of the campaigns, especially those in the interval between the first and second world wars, had some success. In general, however, according to data obtained immediately after the second world war, the prevalence of endemic syphilis had been the same for many previous decades. 


\section{Definition of endemic syphilis}

In his monograph Grin stressed the fact that endemic syphilis in Bosnia was not a clinical, but an epidemiological, problem. ${ }^{6}$ Environmental factors clearly played an important part in its appearance.

According to Grin, ${ }^{6}$ the main characteristics of endemic syphilis in Bosnia were as follows. The propagation of the infection in the family and the community was predominantly by an extragenital route. It had no connection with sexual activity, and was not supposed to be sexually transmitted. The disease was present equally in both sexes, regardless of age. Infection took place in childhood. Standards of hygiene and culture were very low. The clinical manifestations of endemic and sporadic syphilis were identical. All these factors predisposed to the endemic character of the disease.

\section{Epidemiology}

Endemic syphilis in Bosnia existed exclusively in rural areas, but its distribution, even in similar conditions, was not the same everywhere (that is, some villages were infected, others not, even if they were nearby). Many factors, including family relationships, social conditions, the density of the population, and communications, influenced the incidence of the disease.

When the incidence was increasing, many new cases were found in children. Conversely, when the disease was in regression, the incidence in children was lower. ${ }^{7}$

Endemic syphilis in Bosnia had the character of a familial disease, and low cultural and hygienic standards predisposed to its spread. These included indirect extragenital spread, mainly by the use of the same containers, cups, and plates for food and beverages (oral contact), and direct extragenital spread of the infection in newborn babies and children in play groups.

According to the data given by Grin, the ratio of incidence in males to females was $1: 1 \cdot 5$. Certain principles for the spread of infection were also found; an infected father transmitted the disease to his wife, she infected her youngest child, and thereafter the disease spread to the other children or members of the family. In the case of an infected wife, she transmitted the disease to the youngest child, then to the husband and other members of the family. If a child was infected, the father was often either not affected or was the last to acquire the disease.

\section{Clinical findings}

At a dermatology congress in Hamburg in 1921 Alexander Glück was the first author who gave a description of endemic syphilis in Bosnia. ${ }^{8}$ Identical data were subsequently presented by Fleger ${ }^{9-12}$ and by
Grin. In his monograph Grin stated that clinical signs of endemic syphilis in Bosnia were identical to those of sporadic cases. ${ }^{6}$

\section{PRIMARY LESIONS}

Primary lesions were seen rarely. In Bosnian villages the infection occurred by short and repeated extragenital contacts (indirect or direct). The initial sclerotic lesion depended on the size of inoculum or on the conditions under which the infection developed. There was no apparent histological reaction at the site of infection (asymptomatic infection), or if the infection was massive a typical primary lesion could be seen.

\section{SECONDARY LESIONS}

Secondary symptoms were similar in endemic and sporadic syphilis. In some cases intense manifestations of the disease could be seen, especially around the mouth. Sometimes they were the first lesions noted, with no previous sclerotic lesions or exanthema. In general, most $(60 \%)$ of the patients were diagnosed in the latent period of the disease. The field examinations also showed that patients with endemic syphilis might have had all the forms affecting the central nervous system, but most of them had atypical, rudimentary, or stationary forms. ${ }^{13}$ The cardiovascular system and other organs were also affected, but no large scale studies were undertaken.

\section{TERTIAR Y LESIONS}

Tertiary disease in patients with endemic syphilis was identical to that form in sporadic cases, but destructive lesions were even more prominent. These lesions most often affected the skin, upper pharynx, and osteoarticular system. This stage of the disease was seen in patients regardless of age. On the basis of cited epidemiological data and the fact that, in families with recent infections, some members had contagious secondary syphilis and simultaneously, or some weeks later, the other members developed gummatous lesions, ${ }^{8}$ the conclusion was reached that reinfection (in previously infected, but immunologically compromised people) was the main reason for the large number of patients with tertiary syphilis in endemic areas.

\section{CONGENITAL SYPHILIS}

After intense action against syphilis in Bosnia, contrary to long maintained opinion, it has become less certain that the absence of congenital syphilis is one of the chief characteristics of endemic syphilis. Although there are no data on its incidence, polls were conducted to compare the state of healthy and infected populations living under the same conditions. The death rate in children aged under one year born to 
mothers in the endemic region (compared with healthy mothers) amounted to $31 \cdot 8 \%(v 26 \cdot 1 \%)$, the rate of abortions to $6.5 \%(v 6.3 \%)$, and that of stillborn children to $3 \cdot 1(v 1 \cdot 4 \%)$. (The poll was conducted in a limited area.)

\section{APPARENT BENIGNITY}

The apparent benignity changed with changed conditions of life. In other words, with improvements in standards of living and increased social obligations the manifestations of syphilis that had not been important became severe disturbances.

\section{Control programme}

The control of syphilis in Bosnia was organised and started by Professor Ernest Grin in 1948 and lasted until the end of 1951. Professor Grin established the following principles. ${ }^{6}(1)$. The campaign and action must actively and directly work in the field. Systematic serological examinations must be carried out on the whole population of the endemic region and followed by adequate treatment of all diseased people. (2). Health control should be introduced and maintained on the basis of files on families, as the basic epidemiological unit. Individual records ought to be attached to the family files. (3). Serological control must also include the healthy population and be repeated at short intervals. The organised field action must not be interrupted until the epidemiological situation is such as to be controlled by the regular health service. (4). Prophlyaxis must be undertaken to prevent a potential reservoir of infection in the family. (5). The action must arouse enough interest to secure the co-operation of the population.

Teams that worked in the field consisted of eight to 10 members and had their own well supplied serological laboratories. Serology tests were carried out using the methods of Khan and Meinicke. Measurements of reactions for research was carried out in the serological laboratory in Sarajevo, which was the reference laboratory of the World Health Organisation. The average capacity of a field laboratory amounted to about 600 examinations a day. Blood samples arrived regularly on the day that they were taken. Systematic clinical and laboratory examinations were performed in villages where there was infection. Infected people were obliged to undergo treatment, and with rare exceptions there was no need to resort to legal proceedings. The diagnosis was ascertained on the grounds of clinical and laboratory findings. If the findings were negative epidemiological factors were also considered.

\section{Work carried out}

During the three years of the programme in 19 districts where syphilis was endemic 35238 diseased people were treated; 3422 of them were in the early stage (primary and secondary), 1928 had late manifestations, 29881 had latent stage disease, and seven had congenital syphilis. Of 552021 blood samples examined, 438863 were from first and 113158 from follow up examinations. The numbers of infected people varied considerably in individual districts, the average amounting to $8.1 \%$ of the total population examined serologically.

Regardless of the clinical picture, the treatment of endemic syphilis consisted of an oil suspension of penicillin with aluminium monostearate (PAM) 3.64.2 MIU for adults divided into six or seven intramuscular injections of $0.6 \mathrm{MIU}$ every day, or $1.2 \mathrm{MIU}$ every other day. A control group of patients was treated with a combination of PAM and bismuth, a total dose of 1.5 MIU PAM given once only, or a total dose of 3.2 MIU PAM divided into two equal doses. The results of treatment of the two different groups did not differ appreciably.

Treatment was repeated only if, at the follow up examination after 8-12 months or later, the serological finding was unchanged or intensified, a negative serological finding had become positive, or syphilis had passed from the latent to the overt stage. In all these cases the total dose given was equal to the total dose given earlier. The total dose of PAM was increased to $7 \cdot 2 \mathrm{MIU}$ only if seropositivity persisted.

Significant side effects of PAM were not registered. Clinical results of the above treatment were excellent in all forms of endemic syphilis. Serological results depended on the duration of endemic syphilis. In early infections the findings were negative after 15-24 months. In gummatous disease, however, clinical recovery did not correlate with serological changes. In late seroresistant cases 2.4 MIU PAM given on days 1, 2 , and 5 gave good results, and in some early cases the findings were negative after only one injection of 1.2 MIU PAM.

The first follow up examinations were carried out eight months after treatment: in early endemic syphilis $84.5 \%(2892 / 3422)$ of patients were seronegative; in latent syphilis $67 \%(20020 / 29881)$ were seronegative; and $4.3 \%(23737 / 552021)$ of serological tests showed unchanged findings at the same titres.

The prophylactic treatment of exposed children consisted of a single dose of 1.5 MIU PAM.

During 38 months of serological and clinical observations $26 \%$ of patients were treated with one or two courses. The repeated treatment could be postponed for a long time ( 24 months), but the serological and clinical follow up examinations could not because 
that would delay the detection of infectious foci.

\section{Epidemiological success}

For the epidemiological success of the campaign it was essential to reduce the source of infection, which meant that at repeat examinations, particularly in expanding foci, new sources of infection had to be sought not only in the group of treated patients but also in the whole population. At the end of the programme it was established that the remaining sources of untreated (that is, unexamined) people represented a greater danger.

If both treatment and prophylaxis had been carried out, at the follow up examination three years later the incidence of seropositive people would have decreased from $2 \cdot 8 \%$ to $2 \cdot 6 \%$.

Extensive prophylactic treatment of exposed people was also important because of "serological and clinical relapse" or seroresistance occurring in families where the source of infection had remained or new infection had been introduced. It was concluded that the "relapse" depended on the reservoir of infection and that reinfection was the chief cause of the apparent failure of the treatment of early endemic syphilis with an adequate dose of PAM.

\section{Decrease in morbidity and reservoirs of infection}

In expanding foci of infection follow up examinations must be carried out three to four months after every treatment. The third and last examination ought to be carried out 38 months after the first. In the above programme five follow up examinations were performed on the whole population (the last in 1952). The result was complete except for some sporadic seroresistant cases. The reservoir of infection was eradicated, health education improved, and the social conditions of life were changed. The first follow up examination of the whole population was carried out six months later. Seropositive findings were reduced to almost half, and the reservoir to $87.5 \%$. Repeat treatment was given to $26 \%$ of patients. The second follow up examination 14 months after the first showed only $5.7 \%$ of seropositive people. They were given a third treatment, and the remainder were treated prophylactically. The third follow up took place seven months after the second: seropositivity amounted to $1.4 \%$ and the reservoir was almost eradicated.

\section{Evaluation of programme}

The eradication of endemic syphilis in Bosnia was evaluated during 1968 to 1970 . Special methodology in co-operation with the WHO was used, which resulted in a documented scientific picture of the campaign. During the evaluation 9331 samples of blood were examined and the cardiolipin reactions (Venereal Disease Research Laboratory, Wasserman reaction, and Meinicke-Khan reaction as well as the fluorescent treponemal antibody-absorption test were carried out in the laboratory of Dr E I Gree's Institute in Sarajevo referent to the WHO, and at the same time also in the reference laboratory in Copenhagen.

In 12 villages 2358 blood samples were also taken and examined by the Treponema pallidum immobilisation test. All the people in whom the findings were positive were examined clinically in detail, and 35 patients with positive results underwent $x$ ray, internal, and ophthalmic examination and had their cerebrospinal fluid tested. The results obtained from these examinations did not show any appreciable late consequences of endemic syphilis.

A particularly important part of this documented survey of the eradication of endemic syphilis was the work on establishing the persistence of treponemes in the anterior chamber of the eye and in the cerebrospinal fluid. Experimentally inoculated rabbits were also observed for a year, and results were negative.

Today endemic syphilis in Yugoslavia is only a historic fact.

\section{References}

1 Glück L. Über das Alter, den Ursprung und die Bennenung der Syphilis in Bosnien und Herzegowina. Archiv für Dermatologie und Syphilis 1889.

2 Bonetić N. A bout of endemic syphilis at a Croatian seaside. (Morbus Skerljevo). Sušak 1936;5:4-5.

3 Fleger $J$. Origin and age of endemic syphilis in Bosnia and Herzegovina. Messenger of Central Institution of Hygiene. 1930;5:1-6.

4 Dojmi L. Dissemination of endemic syphilis in Bosnia and Hercegovina. Messenger of Central Institution of Hygiene 1934;5:230-9.

5 Dojmi L. Eradication of endemic syphilis in the region of Tuzla in 1927-1928. Serbias Archiv 1929;12:4-7.

6 Grin E. Epidemiology and control of endemic syphilis. Geneva: World Health Organisation, 1953. (WHO Monograph Series No 243.) 1-211.

7 Grin E. Basic problems of endemic syphilis in Bosnia and Hercegovina. Health of people 1945;11-12:40-9.

8 Gluck A. About clinical characteristics of endemic syphilis in Bosnia. Archiv für Dermatologie und Syphilis 1922;138:44-9.

9 Fleger J. Change in cerebrospinal fluid of endemic syphilis in Bosnia and Herzegovina. Review of Medicine 1926;5:12-24.

10 Fleger J. General reason for clinical characteristics of endemic syphilis in Bosnia and Herzegovina. Serbiaś Archiv 1928;5: 17-23.

11 Fleger $\mathrm{J}$. Initial sclerosis in consideration of mechanisms of infection with endemic syphilis in Bosnia and Herzegovina. Review of Medicine 1929;2:34-41.

12 Fleger J. Clinical manifestations of endemic syphilis in Bosnia and Herzegovina. Messenger of Therapy 1933;2:14-8.

$13 \mathrm{Zec}$ N. Neuropsychiatric affects and endemic syphilis in Bosnia and Herzegovina. Acta Med Jugosl 1951;5:1-2. 